\title{
Effect of posterior cortical lesion on two versions of the brightness-discrimination task
}

\author{
ANTHONY C. SANTUCCI and F. ROBERT TREICHLER \\ Kent State University, Kent, Ohio
}

\begin{abstract}
The brightness-discrimination performance, of normal and posterior decorticate rats was measured in two test situations designed to provide different spatial task characteristics. A Y-maze and an open field with otherwise matched stimulus properties provided tests with many or few spatial characteristics, respectively. In contrast to predictions from visuospatial integrative interpretations of lesion consequences, the present results indicated no attenuation of the typical operative impairment when the reduced spatial-cue tasks were employed. Furthermore, this outcome was common to both simple acquisition/retention (Experiment 1) and successive discrimination reversal (Experiment 2) versions of the tasks. Interpretations of the relative contribution of memory or visuospatial integrative deficits to the measured behavioral consequences are discussed.
\end{abstract}

Among alternative views advanced to account for the brightness-discrimination losses exhibited upon removal of the rat's posterior cortex are both memorial and visuospatial interpretations. In support of the former, Meyer (1972) has suggested that a preoperatively established memory is not destroyed by a lesion, but, rather, that access to the stored information is impaired. Braun, Meyer, and Meyer (1966) and Jonason, Lauber, Robbins, Meyer, and Meyer (1970) have provided empirical support for this interpretation by demonstrating that an amphetamine dose that does not affect acquisition of a brightness task in naive posterior decorticates does enhance the relearning of the task in similarly lesioned animals. LeVere and LeVere (1982), in agreement with Meyer, have provided a retrieval-based explanation of the typical brightness-discrimination deficit. They have suggested that injury to a part of the integrative system produces a behavioral impairment wherein the neurological control of behavior is shifted to other noninjured, but less efficient, neural systems. When the organism comes to once again utilize the injured, but still functional, neurological system, performance is restored and recovery is inferred. Apart from the neurological mechanisms, this view implies that the memory is maintained and the behavioral deficit is based on a temporary inability to retrieve the previously stored information.

The authors thank D. C. Riccio and P. Kasenow for their comments on an earlier version of this manuscript. Also, the help of Y. Hines in histology and M. Ryland and C. Velazquez in behavioral testing is gratefully acknowledged. The present research was partially supported by a Grant-in-Aid of Research awarded to the first author by Sigma Xi, The Scientific Research Society, and fulfilled partial requirements for his MA degree at Kent State University. Some of the information in this report was presented at the 1984 meeting of the Midwestern Psychological Association, Chicago. Please direct reprint requests to either author at Department of Psychology, Kent State University, Kent, OH 44242 .
In contrast to memorial interpretations of the lesionbased deficit, Cooper and his co-workers have suggested that an inability to combine visual and spatial components of the criterion task underlies the behavioral deficit associated with posterior decortication. Empirical support for this hypothesis has been provided by Birch, Ferrier, and Cooper (1978), who compared the normal rat's improvement over the course of successive reversals with that of operated animals and noted a significant lesionbased deficit. However, when operated subjects wore translucent, light-diffusing occluders over their eyes, the typical impairment was ameliorated and improvement over successive reversals was observed. Birch et al. contended that occluders reduced the spatial characteristics of the task and allowed subjects to respond to the differential brightness cues without requiring their integration with spatial task components. According to such a visuospatial interpretation, if animals were trained on a brightness discrimination in a situation in which the spatial characteristics of the task were reduced, one might predict some attenuation of the usual lesion-based deficit.

\section{EXPERIMENT 1}

Experiment 1 tested the generality of the consequences of the differential availability of visual and spatial task components by comparing performance on two different devices: the traditional Y-maze, or Thompson-Bryant (1955) task, and an open-field test arena. The open-field variant of the brightness task was used as a means for reducing the marked spatial task properties associated with dual-alternative directional mazes. The rationale for this approach was provided by Becker and Olton (1980), who utilized this test for their studies of hippocampal lesion effects and suggested that the use of multiple locations for placement of discriminanda greatly reduced the probability of reward for a simple directional choice (right vs. 
left). Contrastingly, the Y-maze and similar directional devices maximize the likelihood of reward for spatial choices. Hence, the present design entailed comparisons of pre- and postoperative performance by groups of animals tested in either device.

\section{Method}

Thirty-eight adult (90- to 120-day-old) male Long-Evans hooded rats were maintained at $80 \%$ of their initial body weights via restricted feeding.

Testing was conducted in either an open-field test arena or a Ymaze. Both devices were constructed of 6-mm-thick black acrylic plastic. The open-field enclosed a floor area of $101.5 \mathrm{~cm}^{2}$ with 16 equal $13.5 \times 13.5 \mathrm{~cm}$ squares outlined on the central $55.5 \mathrm{~cm}^{2}$ of this area. Access to the open field was provided by guillotine doors located in the center of each side wall. The Y-maze contained a $26.6-\mathrm{cm}$-long stem alley and two $36.2-\mathrm{cm}$ arms that diverged at a $45^{\circ}$ angle from the end of the alley. These dimensions yielded a distance that was approximately equivalent to the average distance from the entry point to discriminanda placement in the open field. During testing, the Y-maze was placed inside the same arena that contained the open field, and access to the starting arm of the $\mathrm{Y}$-maze was consistently provided through one specific guillotine door in the side wall. Discriminative stimuli were placed on the designated squares or in the ends of the arms when open-field or Y-maze testing, respectively, was being conducted.

After 2 or 3 days of restricted feeding, the animals were put in their assigned training device for an initial $1 / 2 \mathrm{~h}$ of adaptation. On the following day, a pretraining phase was initiated wherein 45 mg Noyes food pellets could be obtained from 4-cm-wide and 6mm-deep food wells on top of $10.1-\mathrm{cm}^{3}$ wooden blocks that were always placed in corners of the field. If an animal failed to consume the pellet within $1 \mathrm{~min}$, it was removed from the device and placed in its home cage in the experimental room for $1 \mathrm{~min}$ before another trial was initiated. On the day after an animal had obtained food within $1 \mathrm{~min}$ on 10 consecutive trials, the requirements of correctional training were introduced. Accordingly, blocks appeared in various locations and pellets were placed in the well of only one block. The subject was required to find the baited block and consume a pellet within $1 \mathrm{~min}$ on 10 consecutive trials.

On the day after the successful completion of pretraining (mean = 4.7 pretraining days), discrimination testing was initiated. Identical $13.5-\mathrm{cm}^{3}$ translucent cubes, which could be illuminated from within, served as discriminanda in both the open-field and Y-maze apparatus. With one cube lighted, these provided values of 270 and $0.8 \mathrm{~cd} / \mathrm{m}^{2}$ for the brighter and dimmer discriminanda, as measured by a Textronic J6511 digital photometer placed $22.5 \mathrm{~cm}$ from the cubes (the average distance from entry doors to discriminanda). Choice of the dimmer cube was always designated correct, and responses were defined by the placement of both forepaws on top of the stimulus cube and, if correct, by consumption of the pellet from that well. Each training session entailed 20 correctional trials, and training was continued until 18 correct initial choices were observed within any 20 consecutive trials. Trials were separated by 10 - to 15 -sec intertrial intervals (ITI), during which the animal was retained in its home cage in the experimental room and the stimuli were rearranged. To minimize potential confounding effects of heat and/or odor cues, the illumination of specific cubes was varied at least once every five trials and the apparatus and objects were washed with a disinfectant between the test sessions of different animals. To eliminate further possible confounding associated with distinctive intertrial activity, similar movements of cubes and food pellets were performed during all ITIs. During open-field testing, a random and counterbalanced sequence provided equal numbers of trials in which the correct object was located near or far from, and right or left of, the entry door. Furthermore, to ensure that the rat's view of the stimuli was not obstructed in the open field, objects were never placed in the two squares nearest and directly adjacent to the entry door. Entrance to the open field was varied systematically, with the four doors used equally frequently over the trial sequence. Selection of squares and arms was done according to a random and counterbalanced sequence with the restriction that no square or arm was correct on more than three consecutive trials. Each subject received one 20-trial test session on each consecutive day throughout the term of testing, with 17 animals being trained in the open field and 21 in the Y-maze.

After original acquisition, approximately half the animals tested in each apparatus ( $n=9$, open field; $n=12, Y$-maze) underwent aspirative lesions of the posterior portion of the neocortex with extents of damage intended to include the areas removed by Jonason et al. (1970) and Treichler and Conley (1982). A 10- to 14-day postoperative recovery period followed surgery; unoperated control subjects ( $n=8$, open field; $n=9$, Y-maze) had a similar period of no testing after completion of original learning. Subsequently, all subjects were tested for retention in the apparatus in which they received their original learning. In the retention test, the subjects were trained until they reattained the 18-correct-within-any-20consecutive-trial criterion.

\section{Results and Discussion}

Both trials and errors to criterion served as dependent variables, but, as Figure 1 illustrates, both measures yielded such similar results that consideration was limited to the trials measure.

The Mann-Whitney U test was used to compare performances of independent groups, and the Wilcoxon $\mathrm{T}$ matched-pairs signed-ranks tests were typically employed to compare outcomes from related groups. Two-tailed tests and an alpha level of .05 were used in all cases.

Because acquisition by control subjects and those scheduled to receive a subsequent lesion were not reliably different in either the open field or $Y$-maze $(U=27$, $p>.05$, and $U=52.5, p>.05$, respectively), acquisi-

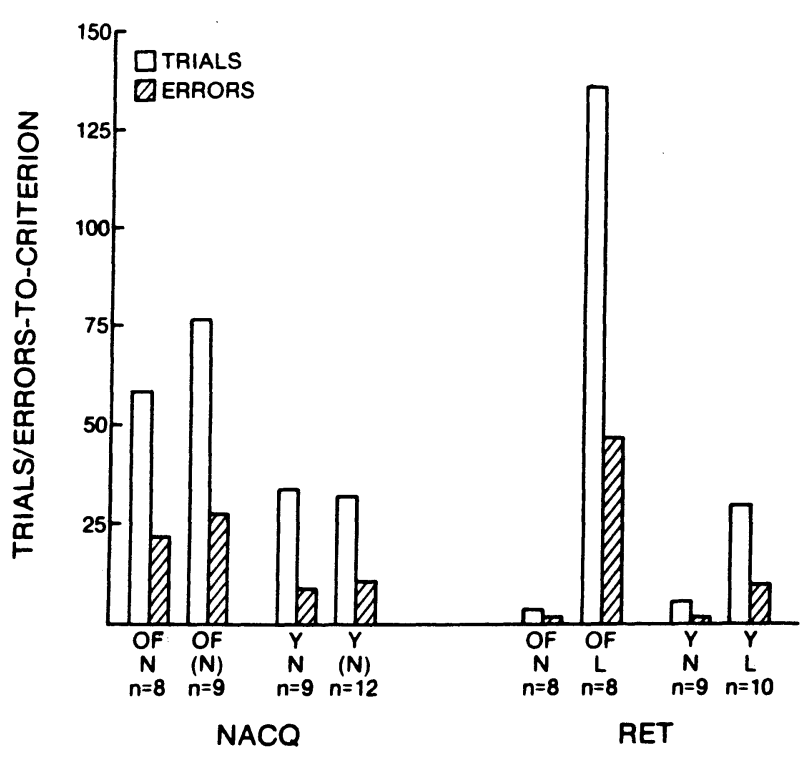

Figure 1. Median normal acquisition (NACQ) and retention (RET) trials and errors to criterion (18 of 20) for animals tested in the open field (OF) and $Y$-maze $(Y) . N=$ normal animals; $(N)=$ normal animals selected to receive postacquisition, posterior decortication; $L=$ posterior decorticates. Note: Subject attrition was due to surgical losses. 
tion scores for all animals tested as normals in a particular device were combined, and performances in the two apparatuses were compared. This comparison yielded trials-to-criterion scores that were significantly greater for original learning in the open field than for original learning in the $Y$-maze $(\mathrm{U}=103.5, \mathrm{z}=2.20, \mathrm{p}<.05)$, and thus the open field was indicated to be the more difficult task.

Retention by unoperated controls was substantial and not different in the two devices $(\mathrm{U}=29.5, \mathrm{p}>.05)$. In both the open field and Y-maze, the median number of trials required by intact animals to relearn the task was less than seven, thus indicating significant savings after original learning $(T=0, p<.05 ; T=5, p<.05$, respectively).

Relearning/retention by operates in the Y-maze required as many trials to criterion as were needed for original acquisition $(\mathrm{T}=22.5, \mathrm{p}>.05)$; relearning in the open field required significantly more trials than were necessary for original learning $(T=3, p<.05)$. Thus, in both test situations, it appeared that further experience was required for the postoperative establishment of criterion performance. However, variability was such that there were no significant differences in the retention scores of normal and brain-damaged subjects tested in the Y-maze $(U=30, p>.05)$, although there were such differences in the scores of those tested in the open field $(U=0$, $p<.05)$. In overview, cortical removals appeared to yield the frequently noted "relearning" impairment, and the impairment was more reliably detected in the more difficult open-field test.

Additional analyses examined performances by the various groups at successively more stringent criteria, from 10 of 20 through 18 of 20 . Figure 2 provides graphic representations of median trials required to meet the various criteria during original learning in the two devices. In addition to the above noted significantly greater scores (at 18 of 20) required for acquisition in the open field, significant differences were also obtained at the 17-of-20 criterion $(U=105, z=2.16, p<.05)$, but at no other,

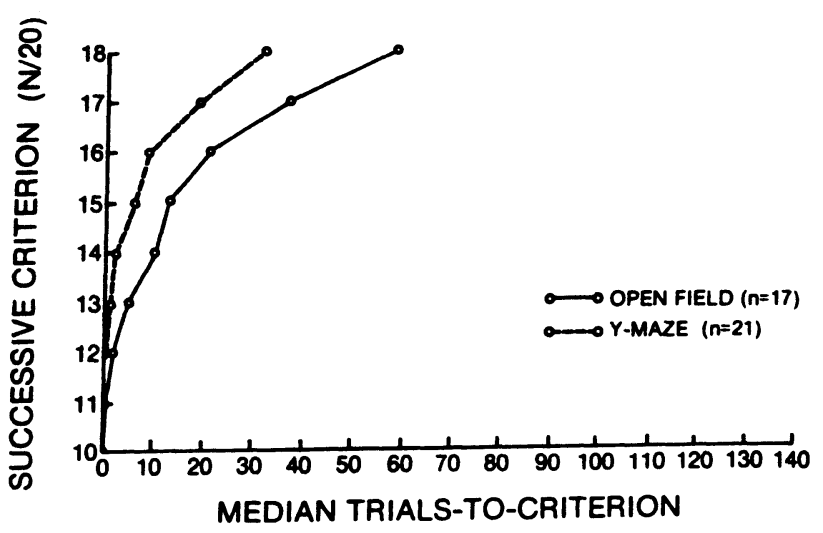

Figure 2. Median normal acquisition trials to criterion required to attain successively more stringent criteria (10 of 20 through 18 of 20) by open-field and Y-maze animals.

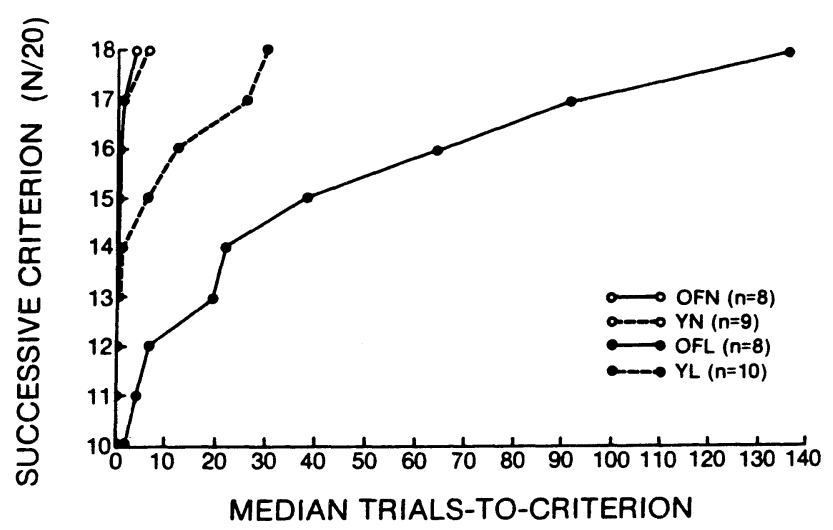

Figure 3. Median retention trials to criterion required to attain successively more stringent criteria (10 of 20 through 18 of 20) by open-field and $Y$-maze normal and lesioned subjects. OFN = openfield normal; YN = Y-maze normal; OFL = open-field lesioned; YL $=$ Y-maze lesioned.

less stringent criteria. Thus, the relatively greater difficulty of the open field seems to be expressed only at the most stern criteria.

Analyses of retention performances associated with the various criteria for the four groups of animals yielded results that were similar to those seen at the 18-of-20 criterion. Figure 3 presents these data graphically.

Normal retention in both devices was characterized by similar, and substantial, nondifferential savings relative to original acquisition. Postoperative retention required significantly more trials in the open field than in the Ymaze at each criterion above 14 of 20 (all Us $<16$, all ps < .05). Because there was much variability in the scores of the open-field lesioned group but the scores of normals tested in the open field were greatly restricted (all near-zero scores), a more conservative statistical test than the Mann-Whitney (Kolmogorov-Smirnov test) was employed to examine differences between these conditions. Lesioned animals tested for retention in the open field required significantly more trials than their corresponding normal relearning group at all criteria greater than 14 of 20 (all $\mathrm{K}_{\mathrm{D} S}=6$, all ps $<.05$ ). In contrast, the lesioned animals tested for retention in the Y-maze did not differ from their controls at any of the successive criteria (all Us $>23$, all ps $>.05$ ).

Pre- and postoperative performances evaluated in the two test situations yielded behavioral deficits that were dissimilar. Although the Y-maze test revealed no difference between original acquisition and postoperative retention at any criteria (all Ts $>5$, all ps $>.05$ ), postoperative retention on the open-field test required significantly more trials than did normal acquisition at all criteria greater than 16 of 20 (all Ts $<4$, all ps $<.05$ ).

\section{EXPERIMENT 2}

Despite the apparent lack of support for the view that reduced spatial task components yield reduced impairment 
on a brightness discrimination, the results of Experiment 1 seemed open to the criticism that the present test technique (acquisition/retention) was different from the one in which Birch et al. (1978) found spatial-cue reduction to be effective in improving performance (successive discrimination reversal, SDR). Experiment 2 attempted to evaluate potential differences associated with these two methods of measuring brightness discrimination. Accordingly, testing in both Y-maze and open field was continued, using subgroups of rats from Experiment 1.

\section{Method}

Training. Four subjects selected at random from each group in Experiment 1 were continued in training on seven SDRs in the same apparatus used for their original testing. All subjects began reversal training immediately upon completion of postoperative relearning. The first reversal required the animal to approach the previously incorrect bright cube to obtain a food pellet. After a criterion of 18 correct out of 20 consecutive trials was reached, the dim object was designated correct and the animal was once again required to reach the 18-of-20 criterion. Redesignation of the correct object continued in this manner until the subject reached criterion on seven successive reversals. If an animal failed to reach criterion within 200 trials on any two reversals, training was terminated and a score of 200 was assigned for that and all further reversals. All other test procedures remained as in Experiment 1.

\section{Results and Discussion}

Figure 4 presents median trials to criterion for each group of subjects at each of the seven reversals. It also provides, for comparison purposes, the median number of trials required by each group to reach the acquisition and retention criteria of Experiment 1 . It should be noted that the acquisition and retention medians are based on only the four subjects that were used in Experiment 2, hence these scores are slightly different from those reported in Experiment 1.

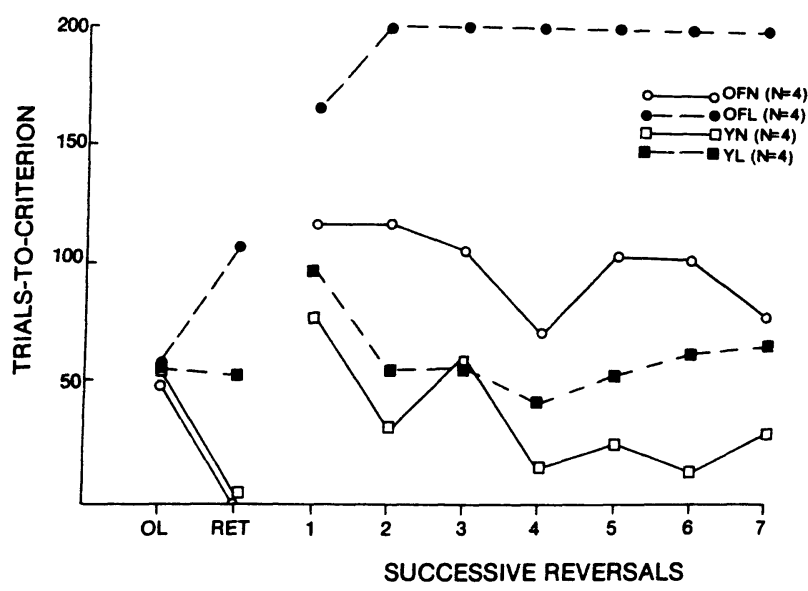

Figure 4. Median normal original learning (OL) and retention (RET) trials-to-criterion from Experiment 1 for the four groups of animals that served in Experiment 2. Median trials to criterion associated with each of the seven successive reversals for all four groups are also presented. OFN = open-field normal; $Y N=$ Y-maze normal; OFL = open-field lesioned; YL = Y-maze lesioned.
Separate Friedman two-way ANOVAs examined performances of various groups of animals over the course of successive reversals. Statistical tests that examined the performances of operates trained in the different devices indicated that open-field subjects exhibited a significant lesion deficit and did not improve over the series of reversals $\left[\chi \mathrm{r}^{2}(6)=2.33, \mathrm{p}>.05\right]$. Because all four animals failed to meet the continuation criterion (18/20 criterion attainment within 200 trials) on at least two of the reversals, their testing was terminated. In contrast, although they did not exhibit significant improvement over the course of training $\left[\chi \mathrm{r}^{2}(6)=11.28, \mathrm{p}>.05\right]$, lesioned animals trained in the Y-maze did attain criterion within the prescribed number of trials on each of the seven reversals. The open-field test, with its presumed property of reduced spatial cues, did not attenuate the typical lesionbased deficit, and, indeed, impairments appeared more severe in the open field than in the Y-maze.

Results from normal subjects trained on SDRs in the two devices were also consistent with the findings from Experiment 1. Significant improvement across reversals was evident only when the performances of Y-maze animals were examined $\left[\chi \mathrm{r}^{2}(6)=19.0, \mathrm{p}<.05\right]$; openfield animals did not show improvement over the course of training $\left[\chi \mathrm{r}^{2}(6)=5.20, \mathrm{p}>.05\right]$. Thus, the openfield tasks seemed also to be more difficult for normal animals tested on SDRs.

In addition, comparisons among the four treatment groups at each reversal were performed using KruskalWallis one-way ANOVAs. These tests yielded significant differences among the groups of subjects at every reversal except the first [all $\mathrm{Hs}(3)>7.8$, all ps $<.05$ ]. Although the small number of animals comprising treatment groups precludes the application of pairwise comparisons, it appeared that the open field yielded a substantial lesion deficit and was difficult for normals. Operated subjects tested in the Y-maze did not improve, but normals showed improvement across the course of reversals.

\section{HISTOLOGY}

After the completion of Experiment 2, all lesioned subjects from Experiments 1 and 2 were perfused intracardially with isotonic saline followed by a $10 \%$ formalin solution. Brains were removed and the extent of damaged cortical tissue in each was plotted on standard Lashley diagrams that were subsequently overlaid by a transparent grid containing 1-mm squares. Scores representative of extent of neurological damage were obtained by counting the number of squares that contained damaged tissue (at least half of the square). The brains were then embedded in celloidin, sectioned at $20-\mu \mathrm{m}$, and stained with cresyl violet. The dorsal lateral geniculate nucleus was examined for the presence of retrograde degeneration, and extent of involved areas was plotted and scored in the same manner as the surface damage. Cortical damage included 
bilateral involvement of nearly the entire posterior half of the six neocortical layers in all animals. Extensive degeneration was observed within the dorsal lateral geniculate nucleus of all subjects, and many showed involvement within the lateral posterior nucleus.

A Mann-Whitney test revealed no significant differences in surface extent of cortical damage for subjects tested in the open field and in the Y-maze on Experiment 1 (U $=28.5, p>$.05). Because some lesioned animals trained in the $Y$-maze $(n=4)$ required less than seven trials for relearning (i.e., less than the median number of trials required by normal retention control animals), a comparison between animals that required less than and greater than seven trials postoperatively was performed to determine whether or not these low scores were associated with limited extents of cortical damage. However, no systematic differences in extent of damage were found between the two groups that exhibited differential relearning scores in the $Y$-maze $(U=9, p>.05)$.

In addition to surface damage, the amount of retrograde thalamic degeneration (dorsal lateral geniculate nucleus) of the animals used in Experiment 1 was also examined. No statistically significant differences were observed between the groups tested in the two devices $(U=33, p$ $>.05$ ), and, as with cortical damage, a comparison between the Y-maze animals that reached retention criterion in less than seven and more than seven trials also revealed no statistically significant differences $(U=11.5, p>$ $.05)$.

Because it was noted that a few animals from Experiment 1 (four from the open field and three from the Ymaze) sustained incidental hippocampal damage, a comparison of postoperative retention performances by animals with or without such damage was also undertaken. These analyses failed to reveal any statistically significant differences in trials to the relearning criterion between these groups of animals (for open field, $U=2$, $\mathrm{p}>.05$; for $\mathrm{Y}$-maze, $\mathrm{U}=10, \mathrm{p}>.05$ ).

Extent of cortical damage and thalamic degeneration in the subgroup of operated animals that were continued on training and tested in Experiment 2 was also examined. No statistically significant differences were observed; open-field and Y-maze subjects sustained equivalent amounts of cortical damage $(U=4, p>.05)$ and thalamic degeneration $(U=4, p>.05)$. Only one of the animals that had sustained hippocampal damage was included in Experiment 2 (in the open-field group), and it appeared that impairment on the successive reversal task could not be attributed to subcortical damage.

The present histological findings suggest that neurological damage was not systematically different among groups of animals that served in either experiment. Hence, variations in tissue destruction did not seem to account for the observed behavioral results.

\section{GENERAL DISCUSSION}

Results of the present investigation failed to extend the generality of the Birch et al. (1978) contention that the postoperative behavioral deficits of striate-lesioned rats are based on an inability to integrate spatial and luminous flux cues. That view has suggested that so long as only luminous flux and not spatial cues are present, lesioned animals show little or no discrimination deficit. However, the present provision of an open-field task designed to reduce the spatial characteristics relative to the usual twoalternative directional maze still yielded impairments at least the equivalent of those seen in a Y-maze with matched discriminative properties. Because the Birch et al. (1978) result had been demonstrated in a test situation in which successive reversals of a brightness task served as the dependent measure, this test procedure was applied in Experiment 2 of the present investigation. Here, too, spatial-cue reduction of the type provided by the open field did not yield any reduction in severity of the lesionbased behavioral deficit, and contrasting outcomes did not seem attributable to inherent differences between successive reversal and simple acquisition measures.

Apart from the present study's failure to support predictions about the consequences of spatial-cue reduction, it did appear that the open-field version of the brightness task provided a distinctive outcome. In both Experiment 1 (acquisition/retention) and Experiment 2 (SDR) postoperative subjects tested in the open field required more trials to criterion than similarly lesioned animals trained in the Y-maze. Thus, although lesions produced similar consequences, detection of reliable differences between performances in the different devices seemed to be based upon the relatively greater difficulty of the open-field task.

Task difficulty as an influence on the magnitude of the lesion deficit in posterior decorticates has previously been noted by Spear and Braun (1969). When their normal and lesioned animals performed a brightness task at intensity ratios of 2 versus $.03 \mathrm{~mL}$ (difficult task) and 75 versus $.04 \mathrm{~mL}$ (easy task) and were trained to a relatively stringent criterion of 20 consecutive correct trials, normal and operated animals showed distinctive consequences of the different intensity ratios. The high-contrast ratio produced only a moderate behavioral impairment, whereas the lesion deficit was exacerbated when the intensities were more similar. A similar consequence of task difficulty was noted by Christensen and Pribram (1979) when they evaluated differences in the consequences of inferotemporal and foveal prestriate lesions in primates.

A slightly different interpretation of the present outcome might be advanced in terms of LeVere and LeVere's (1982) "compensation theory." In keeping with that view, the relatively greater impairment of performance observed when lesioned animals were tested in the open-field brightness task might be attributable to the provision of a greater variety of cues there than are present in a Y-maze. Such open-field properties, in turn, might require elimination of a larger number of alternative solutions before subjects utilized their unimpaired visual capabilities, and thus display the postoperative restitution of performance associated with "compensation theory."

Although the present report does not provide support for a perceptual interpretation of the typical lesion deficit 
exhibited by posterior decorticates, it is compatible with explanations of cortical damage that emphasize inabilities to gain access to or utilize preoperatively stored information. Furthermore, the influence of task difficulty upon behavioral measures of lesion consequences appears to be very similar to that observed in other studies in which access to stored information has been suggested as the basis for impairment. An alternative explanation of results supporting perceptual interpretations of the lesion effect might suggest that the behavioral outcomes are specific to the use of occluders as a way of reducing spatial properties. If so, the generality of the visuospatial integrative interpretation seems somewhat limited.

Because reduction in the spatial properties of a brightness task provided by the present open-field test did not protect against the deleterious effects of cortical removal, the present results do not support the conclusion that an inability to integrate information about brightness and spatial properties underlies the typical lesion-based impairment. Access-to-memory interpretations appear to offer a viable alternative explanation that is consistent with the present results.

\section{REFERENCES}

BeCKer, J. T., \& OLton, D. S. (1980). Object discrimination by rats: The role of frontal and hippocampal systems in retention and reversal. Physiology \& Behavior, 24, 33-38.
Birch, M. P., Ferrier, R. J., \& CoOper, R. M. (1978). Reversal set formation in the visually decorticate rat. Journal of Comparative and Physiological Psychology, 92, 1050-1061.

Braun, J. J., Meyer, P. M., \& Meyer, D. R. (1966). Sparing of a brightness habit in rats following visual decortication. Journal of Comparative and Physiological Psychology, 61, 79-82.

Christensen, C. A., \& Pribram, K. H. (1979). The effect of inferotemporal or foveal prestriate ablation on serial reversal learning in monkeys. Neuropsychologia, 17, 1-9

Jonason, K. R., Lauber, S., Robbins, M. J., Meyer, P. M., \& MEYER, D. R. (1970). Effects of amphetamine upon relearning pattern and black-white discriminations following neocortical lesions in rats. Journal of Comparative and Physiological Psychology, 73, 47-55.

LEVERE, N. D., \& LEVERE, T. E. (1982). Recovery of function after brain damage: Support for the compensation theory of the behavioral deficit. Physiological Psychology, 10, 165-174.

Meyer, D. R. (1972). Access to engrams. American Psychologist, 27, 124-133.

SPEAR, P. D., \& BRAUN, J. J. (1969). Nonequivalence of normal and posteriorly neodecorticated rats on two brightness discrimination problems. Journal of Comparative and Physiological Psychology, 67, 235-239.

Thompson, R., \& BRYANT, J. H. (1955). Memory as affected by activity of the relevant receptor. Psychological Reports, 1, 393-400.

Treichler, F. R., \& Conley, E. J. (1982). Posterior cortical lesion influences on successive discrimination reversal performance by rats. Physiological Psychology, 10, 405-408.

(Manuscript received January 5, 1985; revision accepted for publication June 3,1985 .) 